\title{
Family Transmission of COVID-19 Including a Child with MIS-C and Acute Pancreatitis
}

This article was published in the following Dove Press journal:

International Medical Case Reports Journal

\author{
Maher Abbas (D) \\ Carl-Johan Törnhage (iD) ${ }^{1,2}$ \\ 'Department of Paediatrics, Skaraborg \\ Hospital, Skövde, Sweden; ${ }^{2}$ Department \\ of Paediatrics, Sahlgrenska Academy, \\ University of Gothenburg, Gothenburg, \\ Sweden
}

Introduction: Spread of the novel coronavirus SARS-CoV-2, since at least December 2019, has caused a pandemic. SARS-CoV-2 causes the disease COVID-19, which can affect several human organs. Abdominal pain is one of the known symptoms, but little is known about acute pancreatitis as a complication. As well, knowledge about viral transmission in families is limited. This case report describes MIS-C and acalculous acute pancreatitis in a child who was a member of a family in which four of five members had COVID-19.

Case Report: A previously healthy family was infected by SARS-CoV-2 from an unknown source. The 13-year-old daughter was infected by SARS-CoV-2 and symptomatic during two periods, with an asymptomatic interval in-between. During the first period, she had transient and mild upper respiratory symptoms which was followed four weeks later by a secondary severe illness. At that point, there was inflammation in multiple organs and signs of Multisystem Inflammatory Syndrome in Children (MIS-C) and a Kawasaki-like disease with skin rash, scalded skin in hands and conjunctivitis. Myocarditis, bronchopneumonia, pancreatitis, and hepatopathy without encephalopathy were noted. She required assisted ventilation for 5 days. There were laboratory signs of disseminated intravascular coagulopathy. The multisystem inflammation was treated with intravenous immunoglobulin (IVIG) once a day for four days and immunotherapy (high dose methylprednisolone (IV) once a day, for 12 days, then tapered over 4 weeks, anakinra (IV) four times daily for 12 days), low molecular weight heparin for 22 days and salicylates for 6 weeks leading to full restoration of health. The two brothers and mother in the family had mild to moderate COVID-19 infections. The father was not affected despite close contact with his children. The household transmission and clinical course and outcome are described. No further known COVID-19 infection occurred in the neighborhood during or immediately after the family cluster was discovered.

Conclusion: Penetrance and severity of COVID-19 can vary in family clusters. One adolescent showed a two-phase course with severe infection. This case report highlights MIS-C and acute pancreatitis as a complication associated with COVID-19 in children.

Keywords: children, coronavirus, COVID-19, Kawasaki disease, multisystem inflammatory syndrome in children, MIS-C, pancreatitis, paediatric inflammatory multisystem syndrome, PIMS

\section{Introduction}

Spread of the novel coronavirus SARS-CoV-2, since at least December 2019, has caused a pandemic. ${ }^{1}$ There are great differences in the pattern of the disease and outcome. $^{2-9}$ Age greater than 70 years, cardiovascular-pulmonary disease and diabetes are risk factors. ${ }^{10,11}$ Typical case presentation in children includes respiratory symptoms, hypoxia and to minor degree gastrointestinal symptoms. ${ }^{5,12}$ Severe
Correspondence: Carl-johan Törnhage Department of Paediatrics, Skaraborg Hospital, SE, 54I85, Skövde, Sweden

Tel +46-720 345175

Fax +46-500 478356

Email carl-johan.tornhage@vgregion.se
International Medical Case Reports Journal 2021:14 55-65 
outcomes for children include cerebral stroke, hyperinflammation, cardiovascular illness, a Kawasaki-like disease, and death. ${ }^{13-17}$ Transmission of the virus occurs mostly through coughing and close contact. Droplet transmission through contact with surfaces touched by an infected individual can occur to a minor degree. ${ }^{18}$ Viral inoculation may occur by touching eyes, nose or throat with contaminated fingers. The most important symptoms linked to increased risk of disease transmission are fever, but not cough, fatigue or myalgia. ${ }^{19}$ Children seem to have lower viral loads and milder disease than adults and therefore ought to transmit the virus less. , 4,18,20,21 $^{\text {Younger }}$ children and those with comorbidities may have higher risk for severe COVID-19.,20

Household transmission of COVID-19 has been studied, with differing results. Adults seem to be more susceptible to COVID-19 than children. ${ }^{22,23}$ There have been few studies on children. There seems to be comparatively less transmission from children as index case to adults, as opposed to from adult to child. ${ }^{24}$ The clinical spectrum of COVID-19 varies and includes Paediatric Inflammatory Multisystem Syndrome (PIMS), Multisystem Inflammatory Syndrome in Children (MIS-C), Multisystem Inflammatory Syndrome in Children and Adolescents with COVID-19 and a Kawasaki-like disease. ${ }^{16,17,25-28}$

The case report, here, discusses the household transmission of SARS-CoV-2 and the clinical course of COVID-19 within 1 family, including laboratory findings.

\section{Case History}

The family consists of two healthy parents originally from the Middle East, in their forties, and three healthy children born in Sweden. The two boys, 14- and 11 years old, and their 13-year-old sister lived together alternating weeks with one parent, who have separated. All children have individual rooms in both the maternal and paternal homes.

On Saturday 4th April 2020, day 0, the siblings were in their mother's house. The oldest boy had a sore throat, headache and stuffy nose. He maintained a temperature of $37^{\circ} \mathrm{C}$ and was free of symptoms after 5 days. Day 2, the mother along with her other two children fell ill. The mother was fatigued, with a fever of $39^{\circ} \mathrm{C}$ and headache. She was symptom free after 2 weeks. The youngest boy had a mild cold for a few days. The sister became fatigued with a headache and temperature of $38^{\circ} \mathrm{C}$, and she was symptom free after 5 days. Four weeks later 8th of May, day 34 , she developed a fever between $39^{\circ} \mathrm{C}$ and $41^{\circ} \mathrm{C}$ and fatigue, at her father's home. Day 37, she developed sore throat, cough, shortness of breath, profuse sweating and diarrhea. Her father was highly exposed to her in her illness; he changed her bedding frequently and ate her food leftovers from exposed dishes and cutlery. Day 40, the daughter awoke with a red rash on her palms, which prompted presentation at the pediatric emergency room. The rash spread to her arms, feet, and knees within an hour of arrival there.

\section{Physical Examination at Arrival in the Emergency Room (ER)}

Vital signs: Blood pressure 87/56 $\mathrm{mmHg}$, heart rate 137 bpm, respiration rate $16 / \mathrm{min}, \mathrm{SpO}_{2} 95 \%$ on room air, temperature $39.1^{\circ} \mathrm{C}$, weight $55,4 \mathrm{~kg}$, height $160,3 \mathrm{~cm}$, and BMI 21,6. General condition: Alert and oriented for time and place, fatigued, with mild respiratory distress.

Positive physical findings: conjunctival injections, bilateral slight eye redness. The mouth and throat: Normal tongue, throat minimally erythematous, dry oral mucosa, dry lips with mild vertical cracking. No lymphadenopathy. Regular tachycardic heart rhythm, and a grade II apical systolic murmur. Equilateral breath sounds, no rales or crackles. Erythematous rash on palms, arms and feet.

Laboratory findings in ER: CRP 255, white blood cells $8.1 \times 10 * 9 / \mathrm{L}$, hemoglobin $117 \mathrm{~g} / \mathrm{L}$, and negative nasopharyngeal swab for PCR SARS-CoV-2.

Treatment, Laboratory Findings, Clinic and Outcome $500 \mathrm{cc}$ sodium chloride IV fluid bolus followed by cefotaxime $2 \mathrm{~g}$ each $8 \mathrm{~h}$ IV and tobramycin $7,5 \mathrm{mg} / \mathrm{kg}$ every $24 \mathrm{~h}$ IV for suspected septicemia.

The night to day 41 , clinical status deteriorated with blood pressure $83 / 38 \mathrm{mmHg}$ and desaturation to $\mathrm{SpO}_{2}$ $94 \%$ on nasal canula $2 \mathrm{~L} / \mathrm{min}$. Significant tenderness was noted upon light abdomen palpation without rebound or guarding. Secondary to suspicion of septic shock of unknown origin she was transferred to the intensive care unit (ICU) where clinical status worsened continuously, with an increased oxygen requirement, tachycardia and hypotension. $\mathrm{SpO}_{2}$ was $85 \%$ on $\mathrm{FIO}_{2} 100 \%$. She was intubated and placed on a ventilator secondary to acute hypoxemic respiratory failure. The clinical assessment was suspected COVID-19 infection complicated with cardiorespiratory failure and the patient was transported to a tertiary level ICU. Day 48, the patient was extubated and placed on High-Flow Nasal Cannula (HFNC) with flow 
rate $25 \mathrm{~L} / \mathrm{min}$ and $\mathrm{FIO}_{2} 30 \%$. Weaning from HFNC occurred on day 50 .

Computer tomography of the abdomen showed multiple slightly enlarged mesenteric lymph nodes, and gland conglomeration in the right fossa. There was moderate to extensive free fluid in the pelvis area, consistent with mesenteric adenitis. MRI of the abdomen was normal. Chest X-ray showed increased interstitial markings bilaterally and a slightly enlarged heart, dilated pulmonary vessels and Kerley-B lines, with suspicion of heart failure. Most basal lung segments were included in the computer tomography of the abdomen with findings of posterior small subpleural infiltration and focal isolated ground glass opacification. Findings were deemed consistent with developing pulmonary edema, or pneumonia.

Echocardiography revealed dilated cardiac chambers and reduced left ventricular systolic function, with EF around $20 \%$ and mild mitral valve insufficiency. Coronary artery morphology was normal and there was no pericardial effusion. NT-proBNP was elevated to $23683 \mathrm{ng} / \mathrm{L}$ and increased to more than $35000 \mathrm{ng} / \mathrm{L}$ before normalization within 13 days. Troponin $\mathrm{T}$ and I were increased. Overall, findings were consistent with myocarditis. Together with mucocutaneous-eye symptoms and high fever this fulfilled criteria for a diagnosis of Kawasaki-like disease.

SARS-CoV-2 IgG was positive and endotracheal aspirate was positive for PCR-SARS-CoV-2, day 1 see Table 1. Day 65, serologies for CMV and EBV were indicative of previous infection. Analysis for $16 \mathrm{~S}$ rRNA gene in blood, a bacteria-specific PCR, was negative.
Blood culture from both central vein catheter and radialis artery were negative. Initially, the patient had anemia, with neutrophilic leukocytosis and lymphocytopenia. Later, mildly elevated serum liver enzymes developed, followed by elevated procalcitonin, D-dimer, fibrinogen, PT, APTT and ferritin. Signs of hyperinflammation and hypercoagulability fulfilled the criteria for MIS-C. ${ }^{25,26}$ The patient also had clinical and laboratory signs of acute

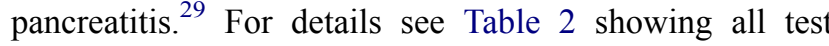
results during hospitalization. Day 65, after two weeks of rehabilitation, she was well without known cardiologic, respiratory, renal, hepatic, pancreatic or neurologic sequelae.

After four months, liver enzymes were normalized. At last control, seven months after onset of COVID-19, her blood cells (including neutrophils and lymphocytes), liver enzymes, bilirubin, pancreas amylase and electrolytes were all normal.

\section{Treatment}

The patient required medical treatment for infection, multisystem inflammatory syndrome and heart failure, in addition to symptomatic treatment and supportive care.

- Treatment of suspected infection:

The first day in the hospital (day 40 of illness) the patient received cefotaxime $2 \mathrm{~g}$ (IV) every $8 \mathrm{~h}$ and tobramycin 7.5 mg (IV) every 24h. Secondary to the patient's critical status, antibiotic treatment was changed to meropenem $1 \mathrm{~g}$ (IV) every $8 \mathrm{~h}$ for 10 days.

Table I PCR for SARS-CoV-2 and SARS CoV-2 IgG Serology

\begin{tabular}{|c|c|c|c|c|c|c|c|c|c|c|c|c|c|c|c|c|c|}
\hline & \multicolumn{6}{|c|}{ Sister } & \multicolumn{3}{|c|}{ Mother } & \multicolumn{2}{|c|}{ Father } & \multicolumn{3}{|c|}{ Brother I } & \multicolumn{3}{|l|}{ Brother 2} \\
\hline & $\begin{array}{l}131 \\
5\end{array}$ & $\begin{array}{l}14 \mid \\
5\end{array}$ & $\begin{array}{l}15 / \\
5\end{array}$ & $\begin{array}{l}20 / \\
5\end{array}$ & $\begin{array}{l}21 / \\
5\end{array}$ & $\begin{array}{l}|7| \\
11\end{array}$ & $\begin{array}{l}15 / \\
5\end{array}$ & $\begin{array}{l}181 \\
5\end{array}$ & $\begin{array}{l}27 I \\
I I\end{array}$ & $\begin{array}{l}18 / \\
5\end{array}$ & $\begin{array}{l}20 / \\
6\end{array}$ & $\begin{array}{l}15 / \\
5\end{array}$ & $\begin{array}{l}161 \\
6\end{array}$ & $\begin{array}{l}|7| \\
I 1\end{array}$ & $15 / 5$ & $\begin{array}{l}161 \\
6\end{array}$ & $\begin{array}{l}171 \\
11\end{array}$ \\
\hline $\begin{array}{l}\text { SARS-CoV-2- RNA } \\
\text { Nasopharynx }\end{array}$ & Neg & Neg & - & $\mathrm{Neg}$ & Neg & - & Pos & Pos & - & $\mathrm{Neg}$ & - & $\mathrm{Neg}$ & - & - & $\begin{array}{l}\text { Not } \\
\text { performed }\end{array}$ & - & - \\
\hline $\begin{array}{l}\text { SARS-CoV-2-RNA } \\
\text { Sputum }\end{array}$ & - & - & Pos & - & - & - & - & - & - & - & - & - & - & - & - & - & - \\
\hline $\begin{array}{l}\text { SARS-CoV-2 lgG } \\
\text { (Architect) }\end{array}$ & - & - & Pos & Pos & - & Neg & - & Pos & Pos & - & $\mathrm{Neg}$ & - & Pos & Pos & - & Pos & Pos \\
\hline $\begin{array}{l}\text { SARS-CoV-2 IgG } \\
\text { AU/ml (iFlash)* }\end{array}$ & - & - & - & 57 & - & 5 & - & - & 52 & - & $\mathrm{Neg}$ & - & 132 & 36 & - & 109 & 31 \\
\hline
\end{tabular}

Note: $* \geq 10=$ Positive 


\begin{tabular}{|c|c|c|c|c|c|c|c|c|c|c|c|c|c|c|c|c|c|c|c|c|}
\hline కి & & & $\bar{\infty}$ & ثै & $\tilde{N}$ & $m_{0}^{m}$ & $\bar{o}$ & $\overline{v_{v}}$ & $\stackrel{\circ}{\circ}$ & $\frac{\infty}{m}$ & $\triangleq$ & $\hat{\mathfrak{N}}$ & & $\frac{8}{v}$ & & & & & $\widetilde{\infty}$ & \\
\hline 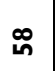 & & & $\stackrel{\llcorner}{\sim}$ & $\stackrel{\sigma}{m}$ & $m$ & $\stackrel{\forall}{0}$ & $\overline{0}$ & $\overline{0}$ & $\stackrel{+}{v}$ & $\frac{\nabla}{\sigma}$ & $\triangleq$ & $\widehat{\sigma}$ & $\begin{array}{l}\hat{2} \\
\text { ô }\end{array}$ & & & & & $\hat{i}$ & $\bar{\sim}$ & \\
\hline in & & & $\stackrel{2}{\sim}$ & $\stackrel{\sigma}{m}$ & $\stackrel{\sim}{N}$ & $\stackrel{m}{0}$ & $\overline{0}$ & $\overline{0}$ & + & 号 & $\hat{\theta}$ & $\hat{\Sigma}$ & $\stackrel{\circ}{\circ}$ & $\frac{8}{v}$ & & & & $\stackrel{\infty}{i}$ & $\stackrel{\circ}{\leftarrow}$ & \\
\hline In & & & & & & & & & in & & & & & & & & & & so & \\
\hline กิ & & & $\stackrel{\underline{2}}{\varrho}$ & 'ं & $\stackrel{\infty}{m}$ & $\stackrel{t}{0}$ & $\overline{0}$ & $\overline{0}$ & o & 号 & $\bar{\varrho}$ & $\underset{\square}{ \pm}$ & $\underline{\sim}$ & & & & & & $\stackrel{\stackrel{n}{2}}{=}$ & \\
\hline กี & & & $\stackrel{\underline{q}}{\varrho}$ & & & & & & $a$ & $\frac{\sigma}{\sigma}$ & 2 & & & & & & & & & \\
\hline $\bar{n}$ & & & 。․ & & & & & & $\underline{m}$ & $\stackrel{\leftrightarrow}{\mathrm{m}}$ & d & $\begin{array}{l}\tilde{\tilde{y}} \\
\underline{\underline{y}}\end{array}$ & $\underline{n}$ & & & & & $\stackrel{\sim}{\sim}$ & $\underset{\sim}{\stackrel{q}{\sim}}$ & \\
\hline in & & & $\stackrel{\forall}{\subseteq}$ & & & & & & $\underline{n}$ & 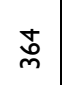 & 은 & $\underset{\sim}{\stackrel{N}{N}}$ & & & & & & $\stackrel{\sim}{N}$ & 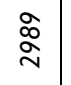 & \\
\hline$\stackrel{\infty}{\sim}$ & & & $\stackrel{\Xi}{\subseteq}$ & 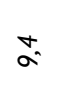 & $\hat{o}$ & đ̃ & $\begin{array}{l}\text { to } \\
0\end{array}$ & 0 & $\stackrel{\circ}{\sim}$ & 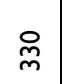 & 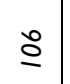 & ঃ & $\stackrel{\infty}{-}$ & $\frac{8}{v}$ & & & & $\stackrel{0}{i}$ & $\frac{O}{d}$ & \\
\hline$f$ & & & $\sigma^{\circ}$ & & & & & & $\forall$ & $\stackrel{\infty}{\sim}$ & & & & & & & & & & \\
\hline ஓீ & & & $\stackrel{\forall}{\stackrel{*}{2}}$ & $\hat{ \pm ే}$ & $\stackrel{\circ}{\circ}$ & $\stackrel{m}{0}$ & $\stackrel{n}{0}$ & 0 & q & స్లి & & $\underset{\sim}{\stackrel{一}{~}}$ & $\stackrel{\infty}{\forall^{\prime}}$ & & & & & & 응 & \\
\hline$\stackrel{\wp}{2}$ & $\times$ & & $\bar{i}$ & & & & & & $\bar{\sigma}$ & $\frac{\sigma}{m}$ & & & & & & & $\stackrel{\infty}{\sim}$ & & \begin{tabular}{l}
8 \\
\multirow{n}{n}{} \\
$\hat{n}^{n}$
\end{tabular} & \\
\hline J & $\times$ & & $\stackrel{m}{\underset{\sim}{d}}$ & & & & & & $\stackrel{ }{=}$ & 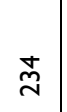 & 2 & ঃ & $\stackrel{a}{\sigma}$ & & $\overline{0^{\circ}}$ & $\stackrel{8}{-}$ & $\hat{i}$ & $\hat{\forall}$ & $\begin{array}{l}8 \\
0 \\
\infty \\
\wedge \\
\wedge\end{array}$ & $\stackrel{\circ}{\therefore}$ \\
\hline $\mathscr{q}$ & $x$ & & $\stackrel{0}{i}$ & 今े & $\tilde{n}$ & ř & 0 & 0 & $\alpha$ & $\stackrel{ \pm}{ \pm}$ & & : & & & & & & & \begin{tabular}{l}
8 \\
\multirow{2}{n}{} \\
ñ
\end{tabular} & \\
\hline $\mathcal{F}$ & $x$ & & $\cong$ & & & & & & $\stackrel{?}{=}$ & $\bar{m}$ & & & & & & & $\alpha^{\circ}$ & & $\begin{array}{l}8 \\
0 \\
\text { D. }\end{array}$ & \\
\hline $\bar{\sigma}$ & $\times$ & & $\begin{array}{l}\stackrel{\alpha}{\infty} \\
\stackrel{0}{2}\end{array}$ & $\stackrel{\circ}{=}$ & $\stackrel{m}{a}$ & $\stackrel{t}{0}$ & $\begin{array}{l}\text { ô } \\
\text { o. }\end{array}$ & 0 & $\stackrel{\alpha}{\sigma}$ & $\bar{\beth}$ & $\alpha$ & $\frac{8}{7}$ & $\mathcal{\sim}$ & & & & & $\underset{\forall}{\forall}$ & $\begin{array}{l}\infty \\
\infty \\
\infty \\
\sim \\
\sim\end{array}$ & \\
\hline ㅇ & & & $\stackrel{\sim}{\simeq}$ & $\hat{\mathrm{O}}$ & $=$ & & & $\bar{o}$ & $\stackrel{\tilde{N}}{\hat{n}}$ & & $\stackrel{\circ}{ }$ & & & & & & & & & \\
\hline & & & $\begin{array}{l}0 \\
\frac{0}{1} \\
0 \\
\dot{f}\end{array}$ & $\begin{array}{l}\stackrel{n}{N} \\
\stackrel{1}{\underline{1}}\end{array}$ & $\begin{array}{l}\infty \\
\stackrel{ \pm}{ \pm} \\
=\end{array}$ & $\frac{0}{\frac{1}{0}}$ & $\begin{array}{l}0 \\
0 \\
0 \\
0 \\
0\end{array}$ & $\begin{array}{l}n \\
0 \\
1 \\
0 \\
0\end{array}$ & $\mathfrak{v}$ & $\begin{array}{l}0 \\
\stackrel{0}{0} \\
\vdots \\
0 \\
\underline{n}\end{array}$ & $\begin{array}{l}\frac{8}{1} \\
\underline{0}\end{array}$ & $\begin{array}{l}\text { ठ্ণ } \\
\text { ٍे }\end{array}$ & $\begin{array}{l}q \\
q \\
o \\
0\end{array}$ & $\frac{8}{v}$ & $\stackrel{\imath}{v}$ & $\begin{array}{l}8 \\
0 \\
0 \\
1 \\
\end{array}$ & $\begin{array}{l}n \\
0 \\
0 \\
v\end{array}$ & $\begin{array}{l}\infty \\
\stackrel{\infty}{0} \\
\infty \\
-\end{array}$ & $\frac{\circ}{\mathrm{n}}$ & $\begin{array}{l}\stackrel{n}{\circ} \\
\text { î }\end{array}$ \\
\hline 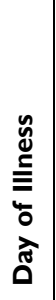 & 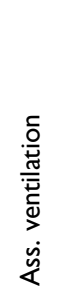 & 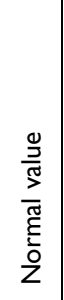 & 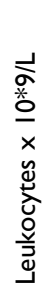 & 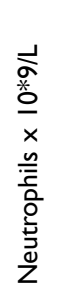 & 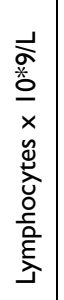 & 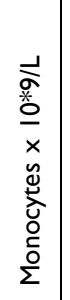 & 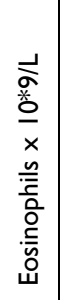 & 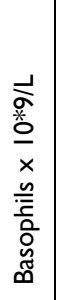 & 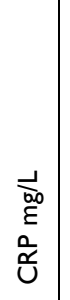 & 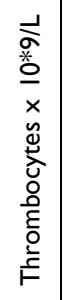 & $\begin{array}{l}\overrightarrow{1} \\
\text { o. } \\
\text { 오 }\end{array}$ & 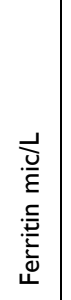 & 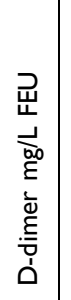 & 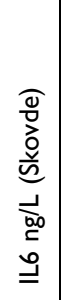 & 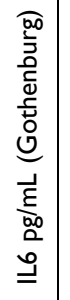 & 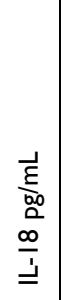 & 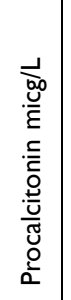 & 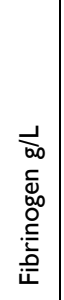 & 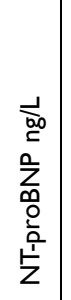 & 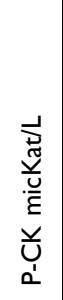 \\
\hline
\end{tabular}




\begin{tabular}{|c|c|c|c|c|c|c|c|c|c|c|c|c|c|c|c|c|c|c|}
\hline & & & $\stackrel{t}{0}$ & $\stackrel{m}{=}$ & $\bar{\sim}$ & $\stackrel{0}{-}$ & & 으 & & å & & $\stackrel{\Xi}{\Xi}$ & & & & & & \\
\hline & & & & & & & & & & & & $\stackrel{n}{\sim}$ & & & & a & & \\
\hline & & & $\begin{array}{l}\omega_{0}^{\prime} \\
\infty\end{array}$ & $\stackrel{\infty}{\sim}$ & $\stackrel{+}{i}$ & & & $\infty$ & & & & $\frac{\infty}{m^{2}}$ & & & & m & & \\
\hline$\stackrel{\circ}{\circ}$ & & & 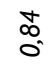 & $\underset{\sim}{\sim}$ & $\stackrel{+}{i}$ & & & $\infty$ & & & & $\stackrel{q}{m}$ & $\hat{\underline{m}}$ & $\stackrel{\infty}{\infty}$ & & & & \\
\hline $\begin{array}{l}\infty \\
: \\
0\end{array}$ & & & $\begin{array}{l}\infty \\
0 \\
0\end{array}$ & 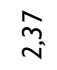 & $\hat{\sim}$ & & & $\infty$ & & & & $\begin{array}{l}\tilde{n} \\
\tilde{m}\end{array}$ & $\stackrel{\circ}{\dddot{2}}$ & $\hat{m}$ & & & & \\
\hline & & & $\begin{array}{l}\partial \\
0\end{array}$ & $\underset{\sim}{\mathbb{N}}$ & $\hat{i}$ & & & $\infty$ & & & & $\begin{array}{l}\infty \\
\stackrel{\infty}{0} \\
i\end{array}$ & & & & & & \\
\hline$\frac{\partial}{\sigma}$ & & & $\stackrel{m}{=}$ & $\underset{\sim}{\sim}$ & $\hat{N}$ & & & $\infty$ & & $=$ & & $\stackrel{\searrow}{\sim}$ & & & & & $\cong$ & นn \\
\hline & & & $\stackrel{0}{0}$ & $\stackrel{\circ}{i}$ & $\stackrel{\sim}{\sim}$ & & & $\stackrel{ \pm}{\Delta}$ & & & & 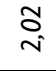 & & & & $\bar{m}$ & & \\
\hline & & & 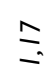 & $\stackrel{\infty}{=}$ & $\cong$ & $\stackrel{m}{\tilde{m}}$ & & $\stackrel{ \pm}{\text { I }}$ & & $\cong$ & & $\stackrel{\infty}{-}$ & & & $\stackrel{\circ}{\sim}$ & & & \\
\hline & & & & & & & & & & & & & & & $\dot{m}$ & & $\cong$ & $\stackrel{\forall}{N}$ \\
\hline & స̃ & & & & & & & & & & & & $\underline{\mathcal{I}}$ & $\stackrel{\Delta}{*}$ & & & \pm & $\stackrel{a}{n}$ \\
\hline & $\stackrel{\text { Ln }}{\stackrel{\sim}{\sim}}$ & & & & & & & & & & & & $\stackrel{\vec{m}}{\underline{m}}$ & $\stackrel{\infty}{m}$ & & $\stackrel{m}{m}$ & 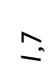 & $\begin{array}{l}\sigma \\
\infty^{-}\end{array}$ \\
\hline & $\alpha^{2}$ & $\bar{m}$ & $=$ & $\bar{\circ}_{0}$ & & & & $\underline{a}$ & $\hat{\sigma^{\circ}}$ & & & & $\stackrel{a}{\underline{m}}$ & $\stackrel{\infty}{m}$ & ํㅜ & 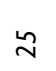 & $\stackrel{\forall}{=}$ & i̊ \\
\hline & & & 葛 & $\underset{\substack{\infty \\
\hdashline \\
0}}{0}$ & ò & & & $\bar{N}$ & $\underline{m}$ & & & & $\stackrel{a}{\underline{m}}$ & $\bar{F}$ & $\hat{f}$ & $m$ & $\underline{n}$ & $\overbrace{0}^{\infty}$ \\
\hline & & $\stackrel{\infty}{\forall}$ & $\underline{\underline{n}}$ & fó & $\stackrel{m}{o}$ & & & $\simeq$ & 으 & $\stackrel{0}{\circ}$ & 아 & & 오 & $\stackrel{m}{q}$ & 6 & $\stackrel{\text { N }}{ }$ & $m$ & $\hat{\infty}$ \\
\hline $\begin{array}{l}a \\
0 \\
0\end{array}$ & & ñ & : & $\hat{0}$ & ò & & $\vec{\sigma}$ & $\hat{N}$ & & & & & $\overline{\underline{m}}$ & $\stackrel{m}{m}$ & & $\dot{m}$ & స్ & $\underline{m}$ \\
\hline & & & & & & & & & & & & กี & $\stackrel{\circ}{\dddot{2}}$ & $\stackrel{+}{m}$ & & $\tilde{m}$ & & \\
\hline $\begin{array}{l}\text { uo } \\
\stackrel{0}{\mathrm{v}}\end{array}$ & $\frac{\nabla}{v}$ & \begin{tabular}{l}
$\simeq$ \\
\multicolumn{1}{c}{} \\
0 \\
0
\end{tabular} & $\hat{\bar{v}}$ & $\stackrel{\circ}{0_{v}}$ & $\begin{array}{l}\hat{\alpha} \\
o \\
\stackrel{0}{-}\end{array}$ & $\begin{array}{l}\frac{0}{0} \\
\frac{1}{0} \\
0\end{array}$ & \begin{tabular}{l}
$m$ \\
\multirow{y}{*}{} \\
$\sigma$
\end{tabular} & $\stackrel{\widetilde{N}}{\mathrm{v}}$ & $\overline{\mathrm{v}}$ & $\stackrel{N}{\mathrm{v}}$ & $\begin{array}{c}\tilde{J} \\
\tilde{N}\end{array}$ & $\frac{\stackrel{1}{0}}{0}$ & $\begin{array}{l}\frac{\underline{v}}{\frac{1}{1}} \\
\underline{m}\end{array}$ & $\begin{array}{l}+\vdots \\
\tilde{y}^{2} \\
m\end{array}$ & $\begin{array}{l}\text { \& } \\
0 \\
\text { d }\end{array}$ & $\begin{array}{l}0 \\
0 \\
0 \\
m\end{array}$ & $\begin{array}{l}\stackrel{w}{m} \\
\stackrel{0}{0} \\
\stackrel{\infty}{0} \\
0\end{array}$ & 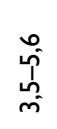 \\
\hline 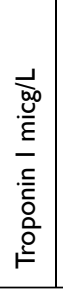 & 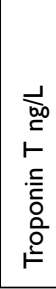 & 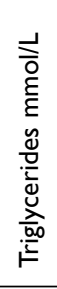 & 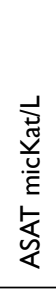 & 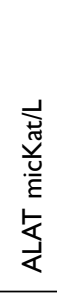 & 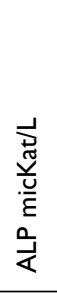 & 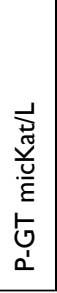 & 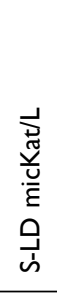 & 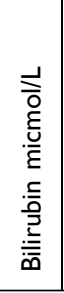 & 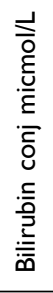 & $\underline{\underline{\alpha}}$ & 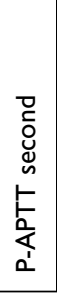 & 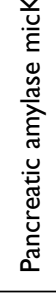 & 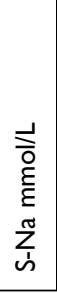 & 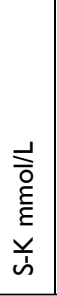 & 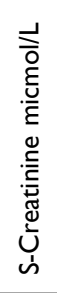 & 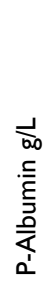 & 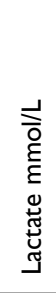 & 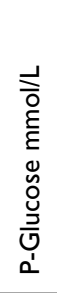 \\
\hline
\end{tabular}




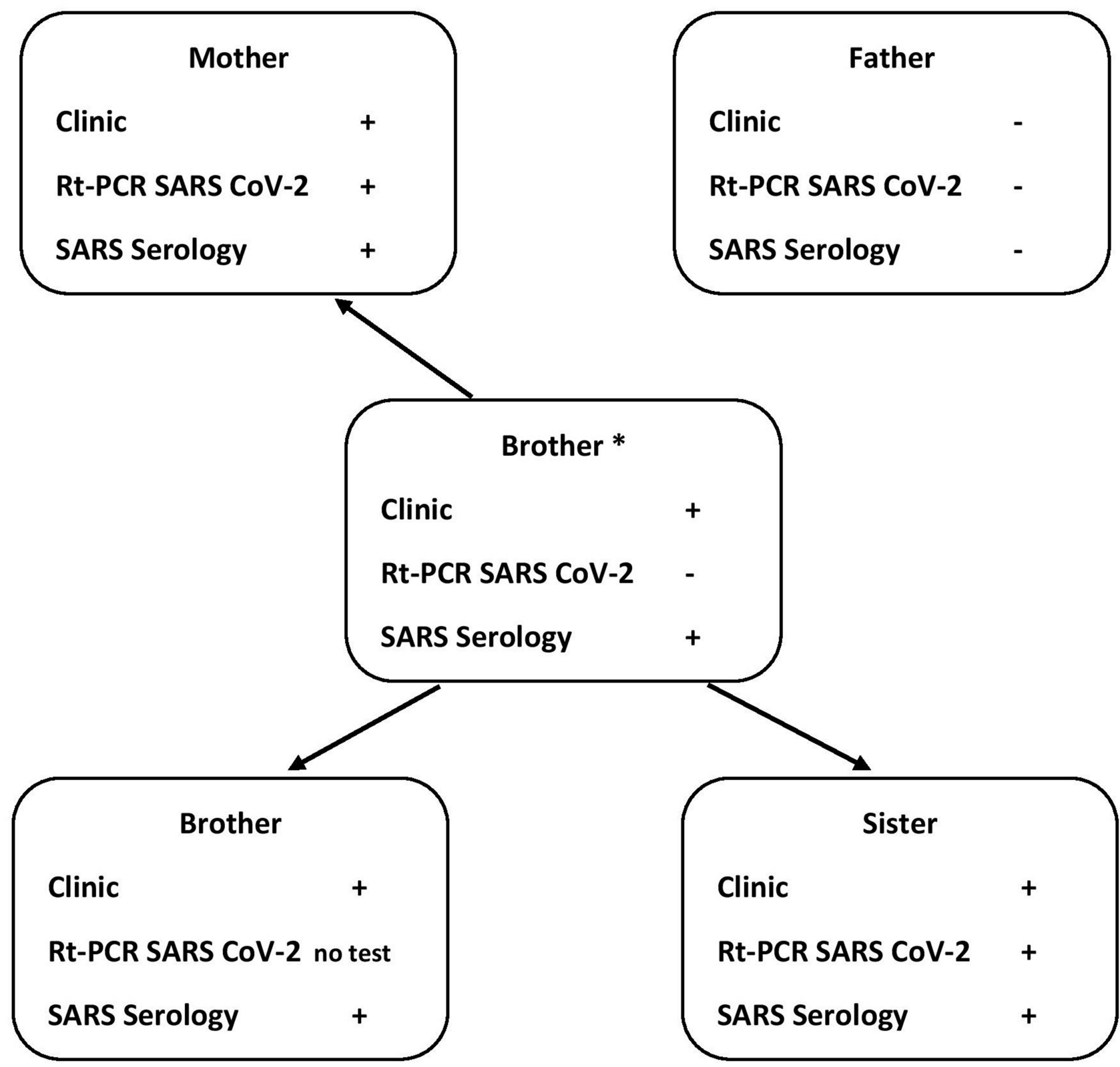

Figure I Household transmission. ${ }^{*}$ Index case.

- Multisystem inflammatory syndrome management from day 41 of illness:

1. Intravenous immunoglobulin (IVIG) $1.5 \mathrm{~g} / \mathrm{kg}$ QD for 2 days, then $1 \mathrm{~g} / \mathrm{kg}$ QD for 2 days (day 41 to 44 of illness).

2. Anakinra $100 \mathrm{mg}$ (IV) four times daily, until the CRP was $<10 \mathrm{mg} / \mathrm{L}$ (day 41 to 52 of illness).

3. Methylprednisolone $60 \mathrm{mg}$ (IV) QD, for 12 days (day 41 to 52 of illness) then tapering over 4 weeks.
4. LMWH (Dalteparin ${ }^{\mathrm{TM}}$ ) $5000 \mathrm{IE}$ (SQ) BID, for 14 days, then tapering over 8 days (day 41 to 62 of illness).

5. Salicylates (ASA) $2.5 \mathrm{mg} / \mathrm{kg} / \mathrm{day}$, for 6 weeks. (from day 62 of illness).

- Heart failure treatment: Noradrenaline $200 \mathrm{ng} / \mathrm{kg} / \mathrm{min}$ $\mathrm{IV}$, Milrinone ${ }^{\mathrm{TM}} 0.7 \mathrm{mic} \mathrm{g} / \mathrm{min}$. and Furosemide ${ }^{\mathrm{TM}}$ $10 \mathrm{mg}$ IV as needed.

- The symptomatic treatment and supportive care: Antipyretic, Esomeprazole ${ }^{\mathrm{TM}}$ 
$\mathrm{IV}=$ intravenous; $\mathrm{SQ}=$ subcutaneous; $\mathrm{QD}$, every day; BID, twice daily.

\section{Household Transmission}

The older brother was believed to have transmitted SARS$\mathrm{CoV}-2$ to his younger siblings and his mother during the siblings' stay at their mother's house. For details see Figure 1. All primary infections were mild to moderate severity. Four weeks later, the daughter became very ill with signs/symptoms of a severe infection/inflammation including high fever. Despite this, her father was never infected, even in the face of close and prolonged contact. The patient presented an extensive antibody response and her brothers, with mild clinical symptoms, were deemed to have even stronger SARS-CoV-2 IgG responses. These antibody responses persisted positive/increased in the brothers and mother, but not in the daughter, 7 months after onset of disease.

Case Definition for Multisystem Inflammatory Syndrome in Children (MIS-C) (CDC, USA). ${ }^{25}$

- An individual aged $<21$ years presenting with fever, laboratory evidence of inflammation, and evidence of clinically severe illness requiring hospitalization, with multisystem $(\geq 2)$ organ involvement (cardiac, renal, respiratory, hematologic, gastrointestinal, dermatologic or neurological); AND

- No alternative plausible diagnoses; AND

- Positive for current or recent SARS-CoV-2 infection by RT-PCR, serology, or antigen test; or COVID-19 exposure within the 4 weeks prior to the onset of symptoms.

Fever $\geq 38.0^{\circ} \mathrm{C}$ for $\geq 24$ hours, or report of subjective fever lasting $\geq 24$ hours including, but not limited to, one or more of the following: an elevated C-reactive protein (CRP), erythrocyte sedimentation rate (ESR), fibrinogen, procalcitonin, d-dimer, ferritin, lactic acid dehydrogenase (LDH), or interleukin 6 (IL6), elevated neutrophils, reduced lymphocytes and low albumin.

\section{Additional Comments}

- Some individuals may fulfill full or partial criteria for Kawasaki disease but should be reported if they meet the case definition for MIS-C.

- Consider MIS-C in any pediatric death with evidence of SARS-CoV-2 infection.

\section{Methods for Analysis of SARS-CoV-2 Antibodies}

We have used SARS-CoV-2 IgG (2019-nCoV) iFlash ${ }^{\text {TM }}$ 1800 Chemiluminescence Immunoassay Analyzer (YHLO (Your Health \& Life Option) BioTech, Shenzhen, China) for semi-quantitative analysis and SARS-CoV-2 IgG ARCHITECT $^{\mathrm{TM}} \quad$ Chemiluminescent Microparticle Immunoassay (Abbot, Sligo, Irland) for a qualitative analysis (negative or positive).

iFlash $^{\mathrm{TM}}$ performance: Cut off is $10 \mathrm{AU} / \mathrm{ml}$. A result less than $10 \mathrm{AU} / \mathrm{ml}$ is considered negative for previous exposure to SARS-CoV-2, $\geq 10$ is considered indicative of previous exposure to SARS-CoV-2.

According to the manufacturer, there is no cross reactivity to $\operatorname{IgG}$ for CMV, Mycoplasma pneumoniae, Chlamydia pneumoniae, Rubella, or Epstein Barr Virus viral-capsid antigen or nuclear antigen. The manufacturer-reported sensitivity is $97.3 \%$ and specificity is $96.3 \%$.

ARCHITECT $^{\mathrm{TM}}$ performance: Cut-off Index $\mathrm{S} / \mathrm{C}$ is 1.4. Index $\mathrm{S} / \mathrm{C}<1.4$ is considered negative for previous exposure to SARS-CoV-2 and Index $\mathrm{S} / \mathrm{C} \geq 1.4$ is considered indicative of previous exposure to SARS-CoV-2.

There was no/low cross reactivity against CMW, EBV, and Herpes Simplex virus. Positive predictive value was dependent on days of symptoms, after 3 to 7 days $25 \%$, after $8-13$ days $83 \%$ and after $\geq 14$ days $100 \%$. Negative predictive value was $99 \%$.

\section{Discussion}

\section{Household Transmission}

This case report of COVID-19 presents a family of two households in which three siblings lived closely together. All siblings and their mother had clinical and laboratory confirmed COVID-19. They had different clinical symptoms and severities. The siblings lived together alternating each week with their mother or father. The parents did not meet each other. When the daughter with COVID-19 lived in the father's household she had a critical immune reaction with high fever $\left(>39^{\circ} \mathrm{C}\right)$ and multisystem effects. During this secondary phase, it seems as if viral transmission was limited and therefore, she did not infect her father, who was always healthy and without PCR or serologic findings of a transient COVID-19 infection. In contrast, the mother, who was not living together with the daughter when she was critically ill, had previous minor clinical symptoms. The mother's PCR and serology were positive most likely because of virus transmission 
from the older son. In the acute period, the older son was PCR SARS-CoV-2 negative and the younger brother was not tested. In retrospect, the older brother is deemed as index, with transmission to the mother and siblings when they all lived together in the mother's house. There is no obvious reason as to why the father was not infected. Perhaps it was because the children had no direct contact with him during the first week of illness with mild symptoms and that there was only a short time window for viral transmission then. This was also suggested by reports by Luo and Ludvigson. ${ }^{18,19}$ Other studies of household transmission show low transmission from children to adults. ${ }^{30}$ A few reports of family clusters in which a child was identified with COVID-19 first, followed by other family members who tested positive, are described by Cao and Zhang. ${ }^{2,15}$ It seems as if children may have a lower viral load. In this case report, the critically ill daughter had a secondary phase with high fever when living together with her father and brothers, followed by a dramatic multisystem inflammatory reaction. During this latter period, she was not deemed to be a high viral transmitter, and this is the probable reason that she did not infect her father.

In a study by Buonsenso et al studying the seroprevalence of anti-SARS-CoV-2 IgG antibodies in children with household exposition to adults with COVID-19, 55\% of all persons in households were SARS-CoV-2 IgG positive. There was no difference in prevalence between adults and children, independent of age. About one third of SARS-CoV-2 IgG positive household members had previously been diagnosed with COVID-19. ${ }^{31}$ These results were lower than demonstrated in our case family $(80 \%)$.

\section{MIS-C}

The daughter with critical COVID-19 had a two-phase illness over a 4 -week period. The first minor illness was followed by a critical period requiring assisted ventilation as she developed Paediatric Inflammatory Multisystem Syndrome (PIMS) and Multisystem Inflammatory Syndrome in Children (MIS-C). She was then positive for SARS-CoV-2 RT-PCR in sputum but not in nasopharynx, and simultaneously positive for SARS-CoV-2 IgG in serum.

MIS-C is a rare post-COVID-19 infection complication, which in a minority of cases can lead to death. It is more common in children over five years age, in contrast to Kawasaki disease has an opposite age spectrum. It is a consequence of an activation of the cellular immune system followed by a massive cytokine storm. Virus antigen can bind to cells, leading to the release of various cytokines. ${ }^{32}$ Consiglio et al have recently found different cytokine storms in Kawasaki disease and MIS-C. Lymphopenia and CRP were more pronounced at onset of COVID-19 in children, who later develop fulminant MIS-C. They also found different plasma protein patterns in MIS-C and Kawasaki. ${ }^{33}$ This agrees with our case findings of very high CRP, lymphopenia and increased IL6 and IL-18 at onset of COVID-19.

\section{Acalcalous Pancreatitis}

The patient presented acalculous pancreatitis (AP) and hepatopathy during the intensive care period. There are only three previous published reports of acute pancreatitis in association with SARS-CoV-2/COVID-19 infection. Alloway et al recently described a radiologically confirmed case of acute pancreatitis in conjunction with COVID-19 infection in a previously healthy 7-year-old girl presenting with abdominal pain and anorexia. ${ }^{34}$ Stevens et al presented a 10-year-old African American girl with an acute pancreatitis as the initial symptom for COVID-19 associated MIS-C, and resulting in good outcome. ${ }^{35}$ Finally, Samies et al reported three 11-16 years-old children with abdominal pain/anorexia and pancreas lipase elevation in combination with radiological images of pancreatitis, in two of three cases. All cases were SARS CoV-2 RT-PCR positive. ${ }^{36}$ The association between AP and SARS-CoV-2 is not well understood. Viral infections, including the coronavirus family, account for $10 \%$ of idiopathic AP. Angiotensin converting enzyme 2 is the human receptor for coronaviruses and is well expressed in intestinal tissues, including the pancreas, and may therefore be the mechanism for pancreatic injury.${ }^{37,38}$ In children with acute abdominal pain, acute pancreatitis caused by SARS-CoV-2 must be a new differential diagnosis.

\section{Kawasaki Disease}

Our patient also developed disseminated intravascular coagulopathy (DIC) and a Kawasaki-like disease. Echocardiography and blood markers confirmed myocarditis and chamber dysfunction. ECG and echocardiography were normal one month after the critical onset. A previous report on Kawasaki syndrome and COVID-19 was presented by Principi. ${ }^{39}$ This report noted associations with IgM antibodies for Staphylococcus aureus, Streptococcus pyogenes and viruses such as EBV, CMV, 2009 H1N1 pandemic influenza virus, and Human 
coronavirus NL63. Our patient was evaluated for evidence of other infectious diseases with no results indicative of current infection for staphylococcal scalded-skin syndrome (SSSS), Toxic shock syndrome (TSS), streptococcal infection, CMV and EBV.

\section{Clinical Course and Outcome}

A report on COVID-19 "The European multinational, multicenter cohort study" by Götzinger et al described 582 children, median age of 5.0 years, with PCR-confirmed SARSCoV-2 infections during 1-24 April 2020. Of these, 363 $(62 \%)$ individuals were admitted to hospital and $48(8 \%)$ required intensive care. Fifteen $(4 \%)$ received mechanical ventilation for a median duration of 7 days. Significant risk factors for requiring ICU admission were low age $<1$ month, male sex, pre-existing medical conditions and presence of lower respiratory tract infection symptoms/signs at presentation. Case-fatality rate was low, $0.69 \%$. By study end, only $4 \%$ of patients were symptomatic or required respiratory support. ${ }^{9}$ Our patient's good clinical outcome agrees with the tendencies in this report.

At onset of illness, the previous healthy girl in this case became fatigued with a headache and temperature of $38^{\circ} \mathrm{C}$ without respiratory symptoms and recovered becoming free of symptoms after 5 days. Despite lack of risk factors mentioned above at onset of illness, she fulfilled all criteria for MIS-C and required ICU admission and assisted ventilation for a short period one month later. Thereafter, she had neither a long convalescence nor persistent respiratory distress. There is no obvious explanation for her fulminant MIS-C when her brothers did not experience any similar symptoms after COVID-19.

\section{Treatment}

The girl was given treatment for her MIS-C with antiinflammatory and immune-modulating drugs after new recommendations after reports of good effects with intravenous immunoglobulin (IVIG), high dose cortisone and anakinra, without adverse reactions. ${ }^{40-42}$

To prevent thrombosis, she received low molecular weight heparin followed by salicylates, without adverse effects. There were no signs of thrombosis at follow up.

Suspected septicemia was treated with broad-spectrum antibiotics: tobramycin and cefotaxime initially, replaced by meropenem as single therapy later. Antibiotic therapy was discontinued after blood and urine cultures were returned negative. Other microbiology testing, including nucleic acid amplification tests and serology, did not demonstrate evidence for concurrent infection.

Our treatment and clinical experience from this case is in concordance with experiences described in the multinational, multicenter COVID-19 study in Europe by Götzinger and from Italian pediatric emergency departments, reported by Parri et al and De Luca et al during the initial phase of COVID-19 in Italy. $6,7,9$

\section{Methods for Analysis of SARS-CoV-2 lgG} For analysis of SARS-CoV-2 IgG, both a qualitative (ARCHITECT $^{\mathrm{TM}}$ ) and a semi-quantitative method (iFlash), were used to confirm diagnosis. Both are wellestablished methods with high sensitivity and specificity. These tests have little or no cross-reactivity to antibodies against other infectious agents. ${ }^{43-46}$

There was a significant margin of reactivity over cut-off reference value for the semi-quantitative test in all siblings. In the mother, there was a strong positive serologic response (ARCHITECT $^{\mathrm{TM}}$ ) but samples were not analyzed with a semi-quantitative method (iFlash), at onset. Seroprevalence of SARS-CoV-2 IgG antibodies in children and mother at follow up 6 months later showed persistent positivity in the brothers and the mother but negativity in the daughter.

\section{Conclusion}

Three healthy children living together alternating weeks with their healthy parents in two separate household became ill with COVID-19 from an unknown source. The mother, but not the father, was also infected. No further known COVID-19 infection occurred in the neighborhood during or immediately after the family cluster was discovered.

The viral transmission in the maternal household was most likely from the older brother to the mother and other siblings. The daughter developed a two-phase illness with a secondary severe phase requiring assisted ventilation for MIS-C, hypercoagulability, myocarditis, pancreatitis, and hepatitis without any short-term sequelae (after 7 months). However, it is important to monitor with long-term follow-up to identify any later consequences of the MIS-C.

\section{Ethics}

Written informed consent for publication of detailed information was obtained from the patient and parent. There was no requirement for regional ethical approval. 


\section{Acknowledgment}

We acknowledge Michael Toepfer, MD, Unilabs AB

Skövde, Sweden for language and content revision.

\section{Funding}

Financial support from The Research Fund at Skaraborg's Hospital Skövde, Sweden for publication fee.

\section{Disclosure}

The authors report no conflicts of interest in this work.

\section{References}

1. Zhu N, Zhang D, Wang W, et al. A novel coronavirus from patients with pneumonia in China, 2019. N Engl J Med. 2020;382(8):727733. doi:10.1056/NEJMoa2001017

2. Cao Q, Chen Y-C, Chen C-L, et al. SARS-CoV-2 infection in children: transmission dynamics and clinical characteristics. J Formos Med Assoc. 2020;119:670-673. doi:10.1016/j.jfma.2020.02.009

3. Cui Y, Tian M, Huang D, et al. A 55-day-old female infant infected with COVID 19: presenting with pneumonia, liver injury, and heart damage. J Infect Dis. 2020;221(11):1775-1781. doi:10.1093/infdis/ jiaa113

4. Dong Y, Mo XI, Hu Y, et al. Epidemiological characteristics of 2143 pediatric patients with 2019 coronavirus disease in China. Pediatrics. 2020;145. doi:10.1542/peds.2020-0702

5. Lu X, Zhang L, Du H, et al. SARS-CoV-2 infection in children. $N$ Engl J Med. 2020;382(17):1663-1665. doi:10.1056/NEJMc2005073

6. De Luca CD, Esposito E, Cristiani L, et al. Covid-19 in children: a brief overview after three months experience. Paediatr Respir Rev. 2020;35:9-14. doi:10.1016/j.prrv.2020.05.006

7. Parri N, Lenge M, Cantoni B, et al. COVID-19 in 17 Italian pediatric emergency departments. Pediatrics. 2020;146(6):e20201235. doi:10.1542/peds.2020-1235.

8. Antunez-Montes OY, Escamilla MI, Figueroa-Uribe AF, et al. COVID-19 and multisystem inflammatory syndrome in Latin American children. Multinational Study Pediatr Infect Dis J. 2020; Publish Ahead of Print. doi:10.1097/INF.0000000000002949.

9. Götzinger F, Santiago- Garcia B, Noguera-Julian A, et al. COVID-19 in children and adolescents in Europe: a multinational, multicentre cohort study. Lancet Child Adolesc Health. 2020;4(9):653-661. doi:10.1016/S2352-4642(20)30177-2.

10. Huang C, Wang Y, Li X, et al. Clinical features of patients infected with 2019 novel coronavirus in Wuhan. China Lancet. 2020;395:497-506. doi:10.1016/S0140-6736(20)30183-5

11. Shi Q, Zhang X, Jiang F, et al. Clinical characteristics and risk factors for mortality of COVID-19 patients with diabetes in Wuhan, China: a two-center, retrospective study. Diab Care. 2020;43(7):1382-1391. doi: $10.2337 / \mathrm{dc} 20-0598$

12. Cai J, Xu J, Lin D, et al. A case series of children with 2019 novel coronavirus infection: clinical and epidemiological features. Clin Infect Dis. 2020:ciaa198. doi:10.1093/cid/ciaa198.

13. Riphagen S, Gomez X, Gonzalez-Martinez $\mathrm{C}$, et al. Hyperinflammatory shock in children during COVID-19 pandemic. Lancet. 2020;395(10237):1607-1608. doi:10.1016/S0140-6736(20) 31094-1

14. Zhou F, Yu T, Du R, et al. Clinical course and risk factors for mortality of adult inpatients with COVID-19 in Wuhan, China: a retrospective cohort study. Lancet. 2020;395(10229):1054-1062. doi:10.1016/S0140-6736(20)30566-3
15. Zhang Y. The epidemiological characteristics of an outbreak of 2019 Novel Coronavirus Diseases (COVID-19) - China, 2020. Chinese J Epidemiol. 2020.

16. European Centre for Disease Prevention and Control. Paediatric inflammatory multisystem syndrome and SARS-CoV-2 infection in children-15 May 2020. ECDC. 2020. Stockholm;

17. Royal college of Paediatrics and Child Health $(\mathrm{RCPCH})$; Health Policy team. Guidance paediatric multisystem inflammatory syndrome temporally associated with COVID-19; 2020. Available from: https://www.rcpch.ac.uk/resource/guidance-paediatric-multisys tem-inflammatory-syndrome-temporally-associated-covid-19.

18. Ludvigsson JF. Systematic review of COVID-19 in children shows milder cases and a better prognosis than adults. Acta Paediatr. 2020;109(6):1088-1095. doi:10.1111/apa.15270.

19. Luo L, Liu D, Liao X, et al. Modes of contact and risk of transmission in COVID-19 among close contacts. medRxiv. 2020;03:24.20042606

20. Jones TC, Mühlemann B, Veith T, et al. An analysis of SARS-CoV-2 viral load by patient age. medRxiv. 2020.

21. Ludvigsson JF. Children are unlikely to be the main drivers of the COVID-19 pandemic - a systematic review [published online ahead of print, 2020 May 19]. Acta Paediatr. 2020. doi:10.1111/apa.15371

22. Zhang Z, Xiao T, Wang Y, et al. Early viral clearance and antibody kinetics of COVID-19 among asymptomatic carriers. medRxiv. 2020;2020. doi:10.1101/2020.04.28.20083139

23. Zhu Y, Bloxham CJ, Hulme KD, et al. Children are unlikely to have been the primary source of household SARS-CoV-2 infections. medRxiv. 2020;2020. doi:10.1101/2020.03.26.20044826

24. Liu Y, Yan LM, Wan L, et al. Viral dynamics in mild and severe cases of COVID-19. Lancet Infect Dis. 2020;20(6):656-657. doi:10.1016/ S1473-3099(20)30232-2.

25. Centers for Disease Control and Prevention (CDC). Health advisory. Multisystem inflammatory syndrome in children (MIS-C) associated with coronavirus disease 2019 (COVID-19). 14 maj 2020. https:// emergency.cdc.gov//han/2020/han00432.asp. Accessed January 18, 2021.

26. Whittaker E, Bamford A, Kenny J, et al. Clinical characteristics of 58 children with a pediatric inflammatory multisystem syndrome temporally associated with SARS-CoV-2. JAMA. 2020;324:259. doi:10.1001/jama.2020.10369

27. WHO Definition. World Health Organization (WHO). Multisystem inflammatory syndrome in children and adolescents temporally related to COVID-19. Scientific brief; 2020. Available from: https:// www.who.int/news-room/commentaries/detail/multisystem-inflamma tory-syndrome-in-children-and-adolsecents-with-covid-19.

28. Verdoni L, Mazza A, Gervasoni A, et al. An outbreak of severe Kawasaki-like disease at the Italian epicentre of the SARS-CoV-2 epidemic: an observational cohort study. Lancet. 2020;395 (10239):1771-1778. doi:10.1016/S0140-6736(20)31103-X.

29. Swaroop VS, Whitcomb DC, Grover S Clinical manifestations and diagnosis of acute pancreatitis; Updated 2019. Available from: https://www.uptodate.com/contents/clinical-manifestations-and-diag nosis-of-acute-pancreatitis?search=pancreatitis\&source=search result\&selectedTitle=1 150\&usage_type=default\&display_rank=1. Accessed 2020

30. Choi SH, Kim HW, Kang JM, et al. Epidemiology and clinical features of coronavirus disease 2019 in children. Clin Exp Pediatr. 2020;63(4):125-132. doi:10.3345/cep.2020.00535

31. Buonsenso D, Valentini P, De Rose C, et al. Seroprevalence of antiSARS-CoV-2 IgG antibodies in children with household exposition to adults with COVID-19: preliminary findings. medRxiv. 2020.

32. Buonsenso D, Riitano F, Valentini P. Pediatric inflammatory multisystem syndrome temporally related with SARS-CoV-2: immunological similarities with acute rheumatic fever and toxic shock syndrome. Front Pediatr. 2020;8:1-5. doi:10.3389/ fped.202000574 
33. Consiglio CR, Cotugno N, Sard F, et al. The immunology of multisystem inflammatory syndrome in children with COVID-19. Cell. 2020;183:968-981. doi:10.1016/j.cell.2020.09.016

34. Alloway BC, Yaeger SK, Mazzaccaro RJ, et al. Suspected case of COVID-19-associated pancreatitis in a child. Radiol Case Rep. 2020;15(8):1309-1312. doi:10.1016/j.radcr.2020.06.009.

35. Stevens JP, Brownell JN, Freeman AJ, Bashaw H. COVID-19-associated multisystem inflammatory syndrome in children presenting as acute pancreatitis. JPGN. 2020;71:669-671. doi:10.1097/ MPG.0000000000002860

36. Samies NL, Yarbrough A, Boppana S. Pancreatitis in pediatric patients with COVID-19. JPIDS. 2020;xx:1-3. doi:10.1093/jipds/ piaa125/5930830

37. Li W, Moore MJ, Vasilieva N, et al. Angiotensin-converting enzyme 2 is a functional receptor for the SARS coronavirus. Nature. 2003;426:450-454. doi:10.1038/nature02145

38. Wan Y, Shang J, Graham R, et al. Receptor recognition by the novel coronavirus from Wuhan: an analysis based on decade-long structural studies of SARS coronavirus. $J$ Virol. 2020;94:e00127-20. doi:10.1128/JVI.00127-20

39. Principi N, Rigante D, Esposito S. The role of infection in Kawasaki syndrome. J Infect. 2013;67:1-10. doi:10.1016/j.jinf.2013.04.004

40. Elias MD, McCrindle BW, Larios G, et al. Management of multisystem inflammatory syndrome in children associated with COVID19: a survey from the international kawasaki disease registry. CJC Open. 2020;2:1-9. doi:10.1016/j.cjco.2020.09.004

41. Feldstein LR, Rose EB, Horwitz SM, et al. Multisystem inflammatory syndrome in U.S. children and adolescents. $N$ Engl J Med. 2020;383(4):334-346. doi:10.1056/NEJMoa2021680
42. Nakra NA, D A B, Herrera-Guerra A, et al. Multi-system inflammatory syndrome in children (MIS-C) Following SARS-CoV-2 infection: review of clinical presentation, hypothetical pathogenesis, and proposed management children (Basel). Children. 2020;7(7):69. doi:10.3390/children7070069.

43. Chew KL, Tan SS, Saw S, et al. Clinical evaluation of serological IgG antibody response on the Abbott Architect for established SARSCoV-2 infection [published online ahead of print, 2020 Jun 9]. Clin Microbiol Infect. 2020;26(9):1256.e9-1256.e11. doi:10.1016/j. cmi.2020.05.036

44. Manalac J, Yee J, Calayag K, et al. Evaluation of Abbott anti-SARSCoV-2 CMIA IgG and Euroimmun ELISA IgG/IgA assays in a clinical lab. Clin Chim Acta. 2020;510:687-690. doi:10.1016/j. cca.2020.09.002

45. Plebani M, Padoan A, Negrini D, Carpinteri B, Sciacovelli L. Diagnostic performances and thresholds: the key to harmonization in serological SARS-CoV-2 assays? Clin Chim Acta. 2020;509:1-7. doi:10.1016/j.cca.2020.05.050

46. Mairesse A, Favresse J, Eucher C, et al. High clinical performance and quantitative assessment of antibody kinetics using a dual recognition assay for the detection of SARS-CoV-2 IgM and IgG antibodies [published online ahead of print, 2020 Aug 25]. Clin Biochem. 2020;86:S0009-9120(20)30817-1. doi:10.1016/j.clinbiochem.2020. 08.009

\section{Publish your work in this journal}

The International Medical Case Reports Journal is an international, peer-reviewed open-access journal publishing original case reports from all medical specialties. Previously unpublished medical posters are also accepted relating to any area of clinical or preclinica science. Submissions should not normally exceed 2,000 words or 4 published pages including figures, diagrams and references. The manuscript management system is completely online and includes a very quick and fair peer-review system, which is all easy to use. Visit http://www.dovepress.com/testimonials.php to read real quotes from published authors. 